\title{
Estrutura populacional de Albizia niopoides (Benth.) Burkart e Mimosa hexandra Micheli (Fabaceae) em Chaco brasileiro Population structure of Albizia niopoides (Benth.) Burkart and Mimosa hexandra Micheli (Fabaceae) in the Brazilian Chaco
}

\author{
Mozart Sávio Pires Baptistal', Vivian Almeida Assunção', Elidiene Priscila Selemel', Ângela Lúcia Bagnatori Sartori' \\ Universidade Federal do Mato Grosso do Sul. Campo Grande, Mato Grosso do Sul, Brasil
}

\begin{abstract}
Resumo: $O$ objetivo do presente estudo foi verificar a existência de variação na estrutura populacional de Albizia niopoides (Benth.) Burkart e de Mimosa hexandra Micheli entre e dentro de remanescentes de Chaco florestado. $O$ estudo foi realizado em dois remanescentes no município de Porto Murtinho, Mato Grosso do Sul, Brasil, a partir de duas parcelas de $2.500 \mathrm{~m}^{2}$ em cada remanescente. Para a análise da estrutura populacional, foram registrados a altura e o diâmetro de todos os indivíduos das espécies em estudo. Foi observada diferença na estrutura populacional entre e dentro dos remanescentes avaliados das duas espécies, resultante principalmente da altura dos indivíduos. A prevalência de indivíduos com menor classe de diâmetro evidenciou que a população é autorregenerativa e o ambiente é heterogêneo, devido provavelmente à inundação sazonal, ao relevo com murundus e também à menor luminosidade, fator que contribui para a estruturação da população das duas espécies, uma vez que se trata de uma vegetação florestal.
\end{abstract}

Palavras-chave: Árvores. Fabaceae. Fitossociologia. Floresta estacional.

Abstract: The objective of the present study was to verify the existence of variation in the population structure of Albizia niopoides (Benth.) Burkart and Mimosa hexandra Micheli among and within forested remnants of Chaco. The study was carried out in two forested remnants of Chaco, where four plots of 2,500 $\mathrm{m}^{2}$ each were established, two plots in each remnant forest. For the analysis of the population structure, height and diameter of all individuals of the studied species were recorded. There was a difference in the population structure of Albizia niopoides and Mimosa hexandra in the Chaco flood plain, due mainly to the height of the individuals, both inside and between the remnants. The prevalence of individuals of smaller diameter class showed that the population is self-regenerative and the environment is heterogeneous, probably due to seasonal flooding. Luminosity is possibly a factor that contributes to the structuring of the population of the two species, since they are forest trees.

Keywords: Trees. Fabaceae. Phytosociology. Phenology.

BAPTISTA, M. S. P., V. A. ASSUNÇÃO, E. P. SELEME \& A. L. B. SARTORI, 2017. Estrutura populacional de Albizia niopoides (Benth.) Burkart e Mimosa hexandra Micheli (Fabaceae) em Chaco brasileiro. Boletim do Museu Paraense Emílio Goeldi. Ciências Naturais 12(2): 169-176. Autor para correspondência: Mozart Sávio Pires Baptista. Universidade Federal do Mato Grosso do Sul. Instituto de Biociências. Laboratório de Botânica. Manoel da Costa Lima - Cidade Universitária. Campo Grande, MS, Brasil. CEP 79070-900 (savbio.ufms@gmail.com). Recebido em 01/05/2017

Aprovado em 23/10/2017

Responsabilidade editorial: Fernando da Silva Carvalho Filho

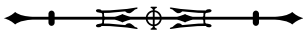




\section{INTRODUÇÃO}

A estrutura populacional de uma vegetação pode ser caracterizada pela variabilidade morfológica e genética dos indivíduos, associada à distribuição da espécie no espaço e no tempo (Hutchings, 1998). Aspectos como taxa de crescimento, mortalidade e recrutamento de espécies podem ser avaliados por meio de fatores bióticos e abióticos (Hutchings, 1998; Hay et al., 2000). A inclusão de fatores abióticos pode auxiliar na descrição da taxa de regeneração (Clark, 1994), em respostas às perturbações (Harper, 1977) e em filtragem ambiental (Cornwell et al., 2006). Parâmetros morfológicos da vegetação podem contribuir para o esclarecimento do estágio sucessional e da produtividade do fragmento, da taxa de recrutamento e de competição (Vanclay, 1994), como observado em Cerrado (Cardoso et al., 2002; Miranda-Melo et al., 2007) e em Restinga (Correia et al., 2010).

O domínio Chaco representa a maior área de floresta seca da América do Sul, com 800.000 km², e abrange quatro países sul-americanos - Argentina, Paraguai, Bolívia e Brasil -, com um setor seco localizado a oeste e outro úmido a leste. No Brasil, setor úmido, o Chaco representa uma pequena área na região sudoeste do Pantanal, em Mato Grosso do Sul (sub-região de Porto Murtinho e Nabileque) (Caputo \& Silva, 2010). Esta região é a única reconhecida como verdadeiramente chaquenha, com 70\% de elementos florísticos de Chaco sensu stricto (Prado, 1993), sendo caracterizada geralmente pela ocorrência de espécies caducifólias, espinescentes e micrófilas (Furtado et al., 1982). Porto Murtinho localiza-se no limite sul do Pantanal (Brasil, 1979) e recebe forte influência da inundação (Eiten, 1983; Fernandes \& Bezerra, 1990; Prado, 1992).

A inundação promove mudanças na estrutura e na composição florística das comunidades presentes no Pantanal (Prado et al., 1994; Schessl, 1999; Damasceno-Junior et al., 2005). Os ecossistemas dessa região são regulados pelo ciclo sazonal de inundações e de secas (Junk et al., 1989), que pode diminuir a taxa de crescimento das árvores (Worbes, 1985), alterar o metabolismo das espécies lenhosas (Joly,
1994) e influenciar na morfologia dos indivíduos, assim como na riqueza, na estrutura e na distribuição das espécies e das comunidades (Junk, 1996). A comunidade vegetal varia ao longo do gradiente de inundação no Pantanal (Cunha \& Junk, 1999, 2001; Silva et al., 2001).

No entanto, não se sabe como a estrutura populacional de espécies de ampla distribuição se organiza diante dos ciclos de inundação do Pantanal. Para isso, é necessário avaliar espécies abundantes na região, e que tenham ampla distribuição, como Albizia niopoides (Benth.) Burkart e Mimosa hexandra Micheli, as quais crescem preferencialmente em matas ciliares e em regiões pantanosas (Carvalho, 2009; Benítez et al., 2013). Elas ocorrem amplamente na América Latina, em distintas vegetações (Pott et al., 2006; Carvalho, 2009). A representatividade pode estar relacionada à dispersão autocórica e anemocórica, e ao longo período de permanência dos frutos na planta-mãe (Carvalho, 2009; Benítez et al., 2013).

Atributos morfológicos, como altura e diâmetro, podem fornecer informações sobre o vigor competitivo, a fecundidade, o tempo de crescimento entre distúrbios, a ocupação do espaço pelo indivíduo, a capacidade de adquirir recursos e a biomassa total (Cornelissen et al., 2003), os quais contribuem para o entendimento da estruturação populacional de uma área em nível de indivíduo. Estudo sobre estruturação populacional no Cerrado demonstrou que os indivíduos dentro do mesmo fragmento apresentaram maior diferença entre si do que entre os indivíduos de outros fragmentos (Miranda-Melo et al., 2007). Trabalhos similares foram desenvolvidos na Venezuela (Ramirez \& Arroyo, 1990), em um arquipélago de ilhas vulcânicas (Milton et al., 1993) e em florestas de altitude (Grau, 2000).

O Chaco de Porto Murtinho, apesar de único no Brasil, é a região mais afetada pelo desmatamento no Pantanal ( $\mathrm{Da}$ Silva et al., 2008), por esta razão são urgentes estudos que tratem sobre a sua conservação. Neste sentido, o objetivo deste trabalho foi avaliar a estrutura das classes de tamanho de duas espécies lenhosas abundantes na região e com ampla 
distribuição, Albizia niopoides e Mimosa hexandra, dentro e entre fragmentos de Chaco florestado. Considerando que essas espécies são adaptadas a ambientes úmidos, espera-se que as populações apresentem maior proporção de indivíduos nas menores classes de tamanho dentro e entre os remanescentes. Este estudo poderá contribuir para a compreensão da organização de populações, ampliando o conhecimento sobre a ecologia do Chaco.

\section{MATERIAL E MÉTODOS}

O estudo foi realizado no município de Porto Murtinho, Mato Grosso do Sul, Brasil, em duas áreas de Chaco florestado, classificadas como Floresta Tropical ou Subtropical Montana Semidecídua, com estrato superior constituído por árvores com até $20 \mathrm{~m}$ de altura. Este estrato é dominado por arbustos adensados, com troncos grossos, profusamente engalhados e espinhosos ou aculeados; o sub-bosque é representado por árvores, arbustos e espécies suculentas (cactos); e o estrato inferior é gramíneo-lenhoso, geralmente descontínuo, com pequena proporção de vegetação herbácea e de epífita xerófita (UNESCO, 1973), equivalendo a Savana Estépica Florestada (Furtado et al., 1982). O clima da região é tropical Aw (Köppen, 1948), com duas estações bem definidas, média pluviométrica anual de $1.282 \mathrm{~mm}$ e temperatura média de $25^{\circ} \mathrm{C}$ (Climate-Data.org, s. d.). A estação seca ocorre de abril a setembro, e a chuvosa vai de outubro a março (Cadavid-Garcia, 1984).

O primeiro fragmento (A1) $\left(21^{\circ} 43^{\prime} 25^{\prime \prime} \mathrm{S}\right.$ e $57^{\circ} 53^{\prime}$ 50,9” W) apresenta estratificação vertical, com árvores de 10 a 15 metros que formam o dossel, predominando-se as espécies arbóreas Schinopsis balansae Engl. (Anacardiaceae), Aspidosperma quebracho-blanco Schltdl. (Apocynaceae), A. triternatum N. Rojas (Apocynaceae) e Tabebuia nodosa (Griseb.) Griseb. (Bignoniaceae). O subdossel é constituído por arbustos e subarbustos de 1-3 m, sendo formado principalmente por Croton spp. (Euphorbiaceae), Zanthoxylum fagara (L.) Sarg. (Rutaceae) e Machaonia brasiliensis (Hoffmanss. ex Humb.) Cham. \& Schltdl.
(Rubiaceae). O solo é coberto por serrapilheira e possui drenagem lenta, permanecendo encharcado durante os meses de maior precipitação (outubro, novembro e fevereiro). O remanescente apresenta sinais de antropização (e.g., pecuária e retirada seletiva de madeira).

O segundo fragmento (A2) (21 42' 19,1" S e $57^{\circ} 45^{\prime} 49,4^{\prime \prime}$ W) apresenta predomínio no dossel de árvores que atingem até $12 \mathrm{~m}$ de altura, sendo constituído por Mimosa hexandra, Aspidosperma quebracho-blanco, Libidibia paraguariensis (D. Parodi) G. P. Lewis e Prosopis rubriflora Hassl. $\bigcirc$ subdossel deste fragmento apresenta arbustos e subarbustos com até $3 \mathrm{~m}$, sendo representado por Croton sp., Machaonia brasiliensis e por indivíduos jovens de Schinopsis balansae. Nesta área, são observados murundus (pequenas elevações do terreno) e a água excedente de um córrego situado nas adjacências do remanescente, devido ao escoamento lento do solo, que promove alagamento.

Em cada fragmento, foi amostrado 0,5 hectare, delimitado por parcelas descontínuas $(50 \times 50 \mathrm{~m})$. Para a análise de estrutura populacional, foram avaliadas a altura (m) e o diâmetro à altura do solo (DAS) (cm). Utilizou-se o teste de Kolmogorov-Smirnov (Massey, 1951) para avaliar as diferenças na distribuição de frequência dos valores de DAS e a altura dentro das áreas de estudo e entre elas. Para verificar a existência de relação entre essas variáveis, foi realizado o teste de Correlação de Spearman (Zar, 1984).

\section{RESULTADOS}

Foram observados 397 indivíduos de Albizia niopoides em A1 e 230 em A2, e 382 indivíduos de Mimosa hexandra em A2, havendo diferença na estrutura de tamanho deles (altura $D_{\text {máx }}=0,48, p<0,001$ e DAS $\left.D_{\text {máx }}=0,66, p<0,001\right)$. A maioria dos indivíduos estava distribuída nas classes inferiores de altura e de DAS em $A$. niopoides (Figuras $1 \mathrm{~A}$ e $2 \mathrm{~A}$ ) e em M. hexandra (Figuras 1B e 2B), com correlação positiva entre altura e DAS em $M$. hexandra $\left(r_{s}=0,88 ; p<0,0001\right)$, e em $A$. niopoides nos dois remanescentes $\left(r_{s}=0,84, p<\right.$ $0,0001 \mathrm{em} \mathrm{A1} ; r_{\mathrm{s}}=0,66, \mathrm{p}<0,0001 \mathrm{em} \mathrm{A2}$ ). 
A estrutura populacional diferiu entre as espécies de acordo com a escala de análise. Entre as áreas, $A$. niopoides mostrou diferença tanto para altura $\left(\mathrm{D}_{\text {máx }}=0,14\right.$, $p<0,005)$ quanto para DAS $\left(D_{\text {máx }}=0,15, p<0,005\right)$. Esta espécie apresentou também variação na estrutura de tamanho entre as áreas, tanto para altura $\left(D_{\text {máx }}=0,43\right.$, $p<0,001)$, quanto para DAS $\left(D_{\text {máx }}=0,58, p<0,001\right)$ em A1. Não houve variação em $A 2$ (altura $D_{\text {máx }}=0,13, p$ $\left.>0,10 ; \mathrm{DAS}_{\text {máx }}=0,03, \mathrm{p}>0,10\right)$. Mimosa hexandra apresentou variação quanto à altura $\left(D_{\text {máx }}=0,05, p<0,05\right)$, mas não em relação ao DAS dos indivíduos $\left(D_{\text {máx }}=\right.$ $0,06, p>0,10)$

Nas duas espécies estudadas, houve maior número de indivíduos distribuídos nas classes inferiores de altura e DAS, caracterizando uma distribuição em 'J' invertido (Figuras 1 e 2). Albizia niopoides apresentou uma diminuição de mais de $85 \%$ no número de indivíduos entre a primeira e a segunda classes de altura, em ambas as áreas, enquanto para os valores de DAS ocorreu redução de mais de 97\% dos indivíduos da classe inferior para a seguinte.

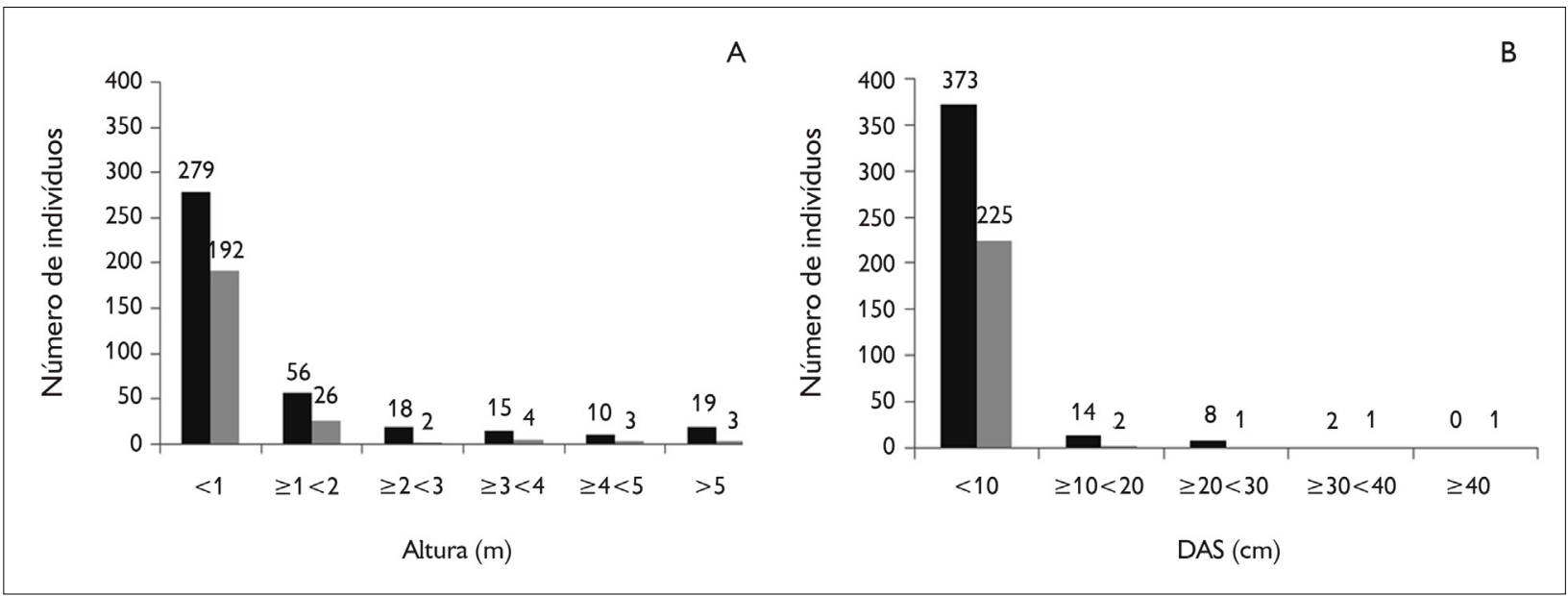

Figura 1. Distribuição da população de Albizia niopoides em classes de altura (m) (A) e diâmetro a altura do solo (DAS) (B) em Chaco florestado, Porto Murtinho, Mato Grosso do Sul, Brasil. A1 está em preto e A2 está em cinza.

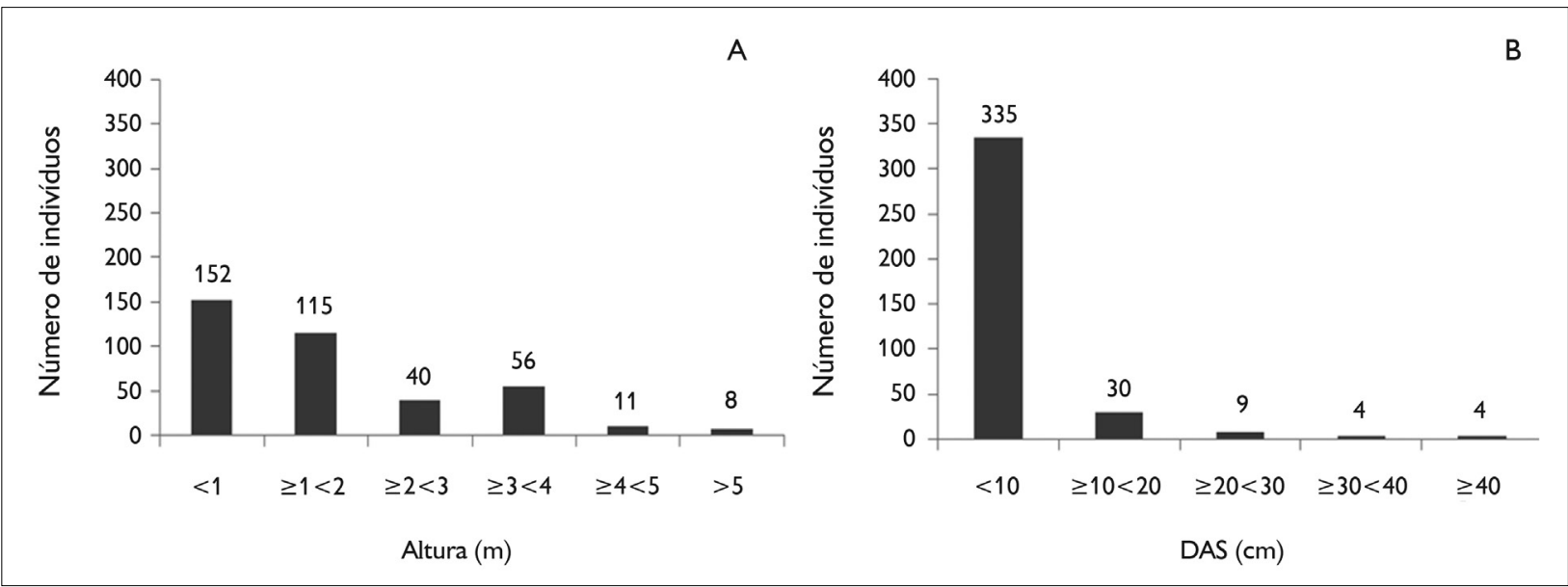

Figura 2. Distribuição da população de Mimosa hexandra em classes de altura (m) (A) e diâmetro a altura do solo (DAS) (B), em Chaco florestado, Porto Murtinho, Mato Grosso do Sul, Brasil.

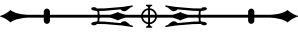




\section{DISCUSSÃO}

O predomínio de indivíduos nas menores classes de tamanho é um indicativo de populações estáveis e autorregenerativas, como observado em remanescentes de floresta na Amazônia (Lopes, 2007) e em Mata Atlântica (Silva et al., 2009). Estudo aponta que a estrutura populacional pode ser influenciada pela heterogeneidade da vegetação e variáveis a ela associadas (Miranda-Melo et al., 2007). A variação observada pode ser uma das razões que favorecem a prevalência dessas espécies nos remanescentes, principalmente em $\mathrm{A} 2$, que, devido à presença de murundus, apresenta maior heterogeneidade do habitat. Quando os murundus ocorrem em áreas sazonalmente inundáveis, funcionam como refúgio para a vegetação contra alagamentos e a saturação hídrica do solo (Oliveira-Filho, 1992).

Diferenças nas taxas de crescimento, obtidas por meio da altura e do DAS, também podem ser correlacionadas com a posição da copa das árvores. As árvores que recebem mais luz demonstram melhor crescimento (Swaine et al., 1987). Em outro trabalho, $A$. niopoides teve índice de Dispersão de Morisita de 1,18 em A1 e 0,98 em A2, o que sugere padrão agregado e regular, respectivamente (Neves et al., 2009). No caso de M. hexandra, é possível que tenha ocorrido diferente investimento da espécie em altura, o que explica a variação encontrada neste parâmetro entre sítios distintos do mesmo remanescente. No entanto, estudos adicionais são necessários para melhor compreensão sobre o padrão de dispersão dessas espécies.

É provável que as diferenças na estrutura populacional de $A$. niopoides e $M$. hexandra estejam relacionadas aos aspectos da biologia dessas espécies, além dos fatores ambientais da área de estudo (murundus e luminosidade). Por exemplo, $A$. niopoides foi predominante em áreas abertas; por se tratar de uma espécie considerada de pioneira a secundária inicial, que não se regenera à sombra, a maior incidência luminosa dessas áreas favorece o seu estabelecimento (Carvalho, 2009). Este aspecto é um indicativo de que, provavelmente, a variação na composição florística de cada remanescente e a cobertura do dossel podem explicar a diferença encontrada na estrutura populacional de $A$. niopoides nos diferentes remanescentes.

Tanto os indivíduos de $A$. niopoides quanto os de M. hexandra mostraram correlação positiva entre altura e DAS. Fatores que regulam a variação de tamanho dos indivíduos podem ser determinantes para a estrutura de uma população (Hutchings, 1998), por exemplo a diferença no tamanho da semente, a taxa de crescimento entre os indivíduos, o tempo de germinação de cada indivíduo em relação aos seus vizinhos e a distância de cada planta em relação aos seus vizinhos mais próximos. Esse aspecto, associado à diferença entre os indivíduos na mesma população, é atribuível a uma mistura entre variabilidade genética e fatores ambientais (Swaine et al., 1987). A maioria dos estudos genéticos realizados com espécies arbóreas (tanto tropicais como temperadas) tem revelado que a maior parte da variabilidade genética se encontra dentro das populações (Hamrick \& Godt, 1990). Geralmente, a divergência genética entre populações não supera 5\% (Sebbenn et al., 2001).

Os resultados indicam que a correlação entre altura e DAS pode estar relacionada à baixa variabilidade genética dos indivíduos. $O$ estudo da estruturação genética em microescala em populações de algumas espécies de Fabacae no Cerrado tem apontado forte endogamia entre as populações e efeito fundador, entre elas Hymenaea stigonocarpa Mart. ex Hayne (Moraes et al., 2007; Moreno et al., 2009), Dipteryx alata Vogel (Soares et al., 2008; Tarazi et al., 2010) e Copaifera langsdorffii Desf. (Tarazi, 2009). O efeito fundador está vinculado à forma de dispersão autocórica dessas espécies (Carvalho, 2009; Benítez et al., 2013). Secundariamente, também ocorre anemocoria em A. niopoides e hidrocoria em M. hexandra. Esse conjunto de síndromes de dispersão promove alto recrutamento de indivíduos com baixa variabilidade genética.

Adicionalmente, a maioria dos indivíduos apresenta pouco desenvolvimento, indicando que não há investimento desproporcional em apenas um dos parâmetros. 
Nesse caso, os indivíduos que possuem maior altura também apresentam maior diâmetro, estando localizados em regiões mais favoráveis, onde a inundação é menos acentuada, sobretudo no fragmento que apresenta maior heterogeneidade, devido à presença de murundus, melhor drenados. Tais aspectos contribuem para corroborar a expectativa de que a maioria dos indivíduos está concentrada nas menores classes de tamanho, devido à sazonalidade hídrica da região, resultante do ciclo de inundação.

\section{CONCLUSÃO}

Diferenças na estrutura populacional de Albizia niopoides e Mimosa hexandra em planície inundável chaquenha foram observadas tanto dentro quanto entre os remanescentes. A prevalência de indivíduos de menor classe de tamanho aponta que a população dessas espécies é autorregenerativa, devido provavelmente à inundação sazonal. Além disso, podemos inferir que o relevo com murundus é outro fator a contribuir para a estruturação da população destas espécies nestas áreas.

\section{AGRADECIMENTOS}

Agradecemos ao Conselho Nacional de Desenvolvimento Científico e Tecnológico (CNPq) (processo n 620176/ 2008-3) e à Fundação de Apoio ao Desenvolvimento do Ensino, Ciência e Tecnologia do Estado de Mato Grosso do Sul (FUNDECT) (chamada n 04/2007), pelo financiamento; à Pós-Graduação em Biologia Vegetal, da Universidade Federal de Mato Grosso do Sul, e aos senhores Sérgio de Oliveira e Leonor, proprietários das fazendas Retiro Conceição e Flores, respectivamente.

\section{REFERÊNCIAS}

BENÍTEZ, B., C. MORALES \& S. BERTONI, 2013. Abundancia de especies forrajeras alternativas en las comunidades naturales en el área de influencia del Arroyo Caañabé. Steviana 5: 89-113.

BRASIL/MINISTÉRIO DO INTERIOR, 1979. Estudo de Desenvolvimento Integrado da Bacia do Alto Paraguai (EDIBAP). Relatório da $1^{a}$ fase: descrição física e recursos naturais. SUDECO, Brasília. Tomo II.
CADAVID-GARCIA, E. A., 1984. O clima no Pantanal matogrossense: 1-39. EMBRAPA-CPAP (Circular Técnica, 14), Corumbá.

CAPUTO, A. C. B. \& J. S. V. SILVA, 2010. Vegetação de Chaco no Pantanal: quanto representa? Embrapa Informática Agropecuária 205-208. Disponível em: <https://ainfo.cnptia.embrapa.br/digital/ bitstream/item/23925/1/p066.pdf>. Acesso em: 15 abril 2017.

CARDOSO, G. C., G. M. ARAÚJO \& S. A. SILVA, 2002. Estrutura e dinâmica de uma população de Mauritia flexuosa L. (Arecaceae) em vereda na estação ecológica de Paga, Uberlândia, MG. Boletim do Herbário Ezechias Paulo Heringer 9: 34-48.

CARVALHO, P. E. R., 2009. Farinha-seca: Albizia niopoides: 1-8. Embrapa Florestas (Comunicado Técnico, 226), Colombo.

CLARK, D. A., 1994. Plant demography. In: L. A. M. C. DADE, K. S. BAWA, H. A. HESPENHEIDE \& G. S. HARTSHORN (Ed.): La Selva: ecology and natural history of a neotropical rain forest: 90-115. The University of Chicago Press, Chicago.

CLIMATE-DATA.ORG, [s. d.]. Clima. Disponível em: <https:// pt.climate-data.org/search/?q=porto + murtinho $>$. Acesso em: 2 agosto 2017.

CORNELISSEN, J. H. C., S. LAVOREL, E. GARNIER, S. DIAZ, N. BUCHMANN, D. E. GURVICH \& J. G. PAUSAS, 2003. A handbook of protocols for standardised and easy measurement of plant functional traits worldwide. Australian Journal of Botany 51(4): 335-380. DOI: <http://dx.doi.org/10.1071/BT02124>.

CORNWELL, E. K., D. W. SCHWILK \& D. D. ACKERLY, 2006. A trait-based test for habitat filtering: convex hull volume. Ecology 87(6): 1465-1471. DOI: <http://dx.doi.org/10.1890/00129658(2006)87[1465:ATTFHF]2.0.CO;2>.

CORREIA, C. M. B., A. T. C. DIAS \& F. R. SCARANO, 2010. Plant-plant associations and population structure of four woody plant species in a patchy coastal vegetation of Southeastern Brazil. Revista Brasileira de Botânica 33(4): 607-613. DOI: <http:// dx.doi.org/10.1590/S0100-84042010000400009>.

CUNHA, C. N. \& W. J. JUNK, 1999. Composição florística de capões e cordilheiras: localização das espécies lenhosas quanto ao gradiente de inundação no Pantanal de Poconé, MT-Brasil. Anais do Simpósio sobre Recursos Naturais e Sócio-econômicos do Pantanal, Manejo e Conservação 2: 387-405.

CUNHA, C. N. \& W. J. JUNK, 2001. Distribution of woody plant communities along the flood gradient in the Pantanal of Poconé, Mato Grosso, Brazil. International Journal of Ecology and Environmental Sciences 27(2): 63-70.

DA SILVA, M. P., R. A. MAURO, M. ABDON, J. SANTOS \& V. DA SILVA, 2008. Estado de conservação do Chaco (Savana Estépica) brasileiro. Anais do Simpósio Nacional Cerrado e Simpósio Internacional Savanas Tropicais 9-2: 1-6. 
DAMASCENO-JUNIOR, G. A., J. SEMIR, F. A. M. SANTOS \& H. F. LEITÃO-FILHO, 2005. Structure, distribution of species and inundation in a riparian forest of Rio Paraguai, Pantanal, Brazil. Flora 200: 119-135. DOI: <http://dx.doi.org/10.1016/j. flora.2004.09.002>.

EITEN, G., 1983. Classificação da vegetação do Brasil: 1-335. CNPq, Brasília.

FERNANDES, A. \& P. BEZERRA, 1990. Estudo fitogeográfico do Brasil: 1-205. Stylus Comunicações, Fortaleza.

FURTADO, P. P., J. O. GUiMARÃES, B. C. FONZAR \& J. M. PIRES, 1982. Vegetação. In: BRASIL. Projeto RADAMBRASIL: Folha SF. 21 Campo Grande: 281-333. Programa de Integração Nacional (Levantamento de Recursos Naturais, 28), Rio de Janeiro.

GRAU, H. R., 2000. Regeneration patterns of Cedrella lilloi (Meliaceae) in Northwestern Argentina subtropical montane forests. Journal Tropical Ecology 16: 227-242.

HAMRICK, J. L. \& M. W. GODT, 1990. Allozyme diversity in plant species. In: A. H. D. BROWN, M. T. CLEGG, A. L. KAHLER \& B. S. WEIR (Ed.): Plant population genetic resources: 43-63. Sinauer, Sunderland.

HARPER, J. L., 1977. Population biology of plants: 1-922. Academic Press, London.

HAY, J. D., M. X. BIZERRIL, A. M. CALOURO, E. M. N. COSTA, A. A. FERREIRA, M. L. A. GASTAL, C. D. GOES-JÚNIOR, D. J. MANZAN, C. R. MARTINS, J. M. G. MONTEIRO, S. A. OLIVEIRA, M. C. M. RODRIGUES, J. A. S. SEYFARTH \& B. M. T. WALTER, 2000. Comparação do padrão da distribuição espacial em escalas diferentes de espécies nativas do cerrado, em Brasília, DF. Revista Brasileira de Botânica 23(3): 341-347. DOI: < http:// dx.doi.org/10.1590/S0100-84042000000300008>.

HUTCHINGS, M. J., 1998. The structure of plant populations. In: M. J. CRAWLEY (Ed.): Plant ecology: 325-358. Blackwell Scientific, Oxford.

JOLY, C. A., 1994. Flooding tolerance: a reinterpretation of Crawford's metabolic theory. Proceedings of the Royal Society of Edinburgh 102: 343-354.

JUNK, W., 1996. Ecology of floodplains - a challenge for tropical limnology. In: F. SHIEMER \& K. T. BOLAND (Ed.): Perspectives in tropical limnology: 255-265. SPB Academic Publishing, Amsterdam.

JUNK, W. J., P. B. BAYLEY \& R. S. SPARKS, 1989. The flood pulse concept in river - floodplain systems. Canadian Special Publication of Fisheries and Aquatic Sciences 106: 110-127.

KÖPPEN, W., 1948. Climatologia: 1-496. Tradução de Guendriss du Klimakunde. Fondo de Cultura Económica, Buenos Aires.
LOPES, M. A., 2007. Population structure of Eschweilera coriacea (DC.) S. A. Mori in forest fragments in eastern Brazilian Amazonia. Revista Brasileira de Botânica 30(3): 509-519. DOI: <http://dx.doi. org/10.1590/S0100-84042007000300015>.

MASSEY, L. H., 1951. The Kolmogorov-Smirnov test for goodness of fit. Journal of the American Statistical Association 46(253): 68-78.

MILTON, S. J., P. G. RYAN, C. L. MOLONEY, J. COOPER \& W. R. J. DEAN, 1993. Disturbance and demography of Phylica arborea (Rhamnaceae) on the Tristan-Gough group of islands. Botanical Journal of Linnean Society 111: 55-70.

MIRANDA-MELO, A. A., F. R. MARTINS \& F. A. M. SANTOS, 2007. Estrutura populacional de Xylopia aromatica (Lam.) Mart. e de Roupala montana Aubl. em fragmentos de cerrado no Estado de São Paulo. Revista Brasileira de Botânica 30(3): 501-507. DOI: < http://dx.doi. org/10.1590/S0100-84042007000300014>.

MORAES, M. L. T., P. Y. KAGEYAMA \& A. M. SEBBENN, 2007. Sistema de reprodução em pequenas populações fragmentadas e em árvores isoladas de Hymenaea stigonocarpa. Scientia Forestalis (74): 75-86.

MORENO, M. A., R. TARAZI, E. M. FERRAZ, F. B. GANDARA \& P. Y. KAGEYAMA, 2009. Estrutura genética espacial em populações de Hymenaea stigonocarpa Mart. ex Hayne mediante a utilização de marcadores moleculares cloroplastidiais. Scientia Forestalis 37(84): 513-523.

NEVES, I. M., E. P. SELEME \& A. L. B. SARTORI, 2009. Distribuição espacial de Albizia niopoides (Leguminosae) em remanescentes de Chaco brasileiro. Revista Brasileira de Agroecologia 4(2): 37103713.

OLIVEIRA-FILHO, A. T., 1992. Floodplain 'murundus' of Central Brazil: evidence for the termite-origin hypothesis. Journal of Tropical Ecology 8(1): 1-19.

POTT, A., V. J. POTT \& T. W. SOUZA, 2006. Plantas daninhas de pastagem na região de Cerrados: 1-336. EMBRAPA Gado de Corte, Campo Grande.

PRADO, D. E., 1992. The Chaco-Pantanal transition in southern Mato Grosso, Brazil. In: J. PROCTOR \& J. A. RATTER (Ed.): Nature and dynamics of forest-savanna boundaries: 51-470. Chapman \& Hall, London.

PRADO, D. E., 1993. What is the Gran Chaco vegetation in South America? I. A review. Contribution to the study of the flora and vegetation of the Chaco. V. Candollea 48(1): 145-172.

PRADO, A. L., C. W. HECKMAN \& F. R. MARTINS, 1994. The seasonal succession of biotic communities in wetlands of the tropical wet-and-dry climatic zone: II. The aquatic macrophyte vegetation in the Pantanal of Mato Grosso, Brazil. Internationale Revue Gesamten Hydrobiologie 79(4): 569-589. DOI: <http://dx.doi.org/10.1002/ iroh.19940790407>. 
RAMIREZ, N. \& M. K. ARROYO, 1990. Estructura poblacional de Copaifera pubiflora Benth. (Leguninosae, Caesalpinoideae) en los Altos Lhanos Centrales de Venezuela. Biotropica 22(2): 124-132.

SCHESSL, M., 1999. Floristic composition and structure of floodplain vegetation in northern Pantanal of Mato Grosso, Brazil. Phyton 39(2): 303-336.

SEBBENN, A. M., C. E. S. SEOANE, P. Y. KAGEYAMA \& C. M. B. LACERDA, 2001. Estrutura genética em populações de Tabebuia cassinoides: implicações para o manejo florestal e a conservação genética. Revista do Instituto Florestal 13(2): 93-113.

SILVA, C. J., K. M. WANTZEN, C. N. CUNHA \& F. A. MACHADO, 2001. Biodiversity in the Pantanal wetland, Brazil. In: B. GOPAL, W. J. JUNK \& J. A. DAVIS (Ed.): Biodiversity in wetlands: assessment, function and conservation: 187-215. Backhuys Publishers, The Netherlands.

SILVA, M. G. C. P. C., A. M. Z. MARTINI \& Q. R. ARAÚJO, 2009. Estrutura populacional de Euterpe edulis Mart. no Sul da Bahia, Brasil. Revista Brasileira de Botânica 32(2): 393-403. DOI: <http://dx.doi.org/10.1590/S0100-84042009000200017>.

SOARES, T. N., L. J. CHAVES, M. P. C. TELLES, J. A. F. DINIZFILHO \& L. V. RESENDE, 2008. Landscape conservation genetics of Dipteryx alata ("baru" tree: Fabaceae) from Cerrado region of central Brazil. Genética 32(1): 9-19. DOI: <https://doi. org/10.1007/s10709-007-9144-7>.
SWAINE, M. D., D. LIEBERMAN \& F. E. PUTZ, 1987. The dynamics of tree populations in tropical forest: a review. Journal of Tropical Ecology 3(4): 359-366.

TARAZI, R., 2009. Diversidade genética, estrutura genética espacial, sistema de reprodução e fluxo gênico em uma população de Copaifera langsdorffii Desf. no Cerrado. Tese (Doutorado em Genética e Melhoramento de Plantas) - Universidade de São Paulo, São Paulo.

TARAZI, R., M. A. MORENO, F. B. GANDARA, E. M. FERRAZ, M. L. T. MORAES, C. C. VINSON \& P. Y. KAGEYAMA, 2010. High levels of genetic differentiation and selfing in the Brazilian cerrado fruit tree Dipteryx alata Vog. (Fabaceae). Genetics and Molecular Biology 33(1): 78-85. DOI: <http://dx.doi.org/10.1590/S141547572010005000007>.

UNITED NATIONS EDUCATIONAL, SCIENTIFIC AND CULTURAL ORGANIZATION (UNESCO), 1973. International classification and mapping of vegetation: 1-99. UNESCO, Paris.

VANCLAY, J. K., 1994. Modelling forest growth and yield: 1-312. CAB International, Wallingord.

WORBES, M., 1985. Structural and other adaptations to long-term flooding by trees in Central Amazonia. Amazoniana 9(3): 459-484.

ZAR, J. H., 1984. Bioestatical analysis: 2. ed.: 1-736. Prentice Hall, New Jersey.

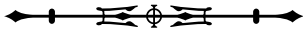

